PHYSICAL REVIEW E 95, 019902(E) (2017)

\title{
Publisher's Note: Regularity criterion for solutions of the three-dimensional Cahn-Hilliard-Navier-Stokes equations and associated computations [Phys. Rev. E 94, 063103 (2016)]
}

John D. Gibbon, Nairita Pal, Anupam Gupta, and Rahul Pandit

(Received 10 January 2017; published 18 January 2017)

DOI: 10.1103/PhysRevE.95.019902

This paper was published online on 12 December 2016 with an error in Eq. (1) and an omission of text after Eq. (2). Equation (1) should read as

$$
\left(\partial_{t}+\boldsymbol{u} \cdot \nabla\right) \boldsymbol{u}=-\nabla P / \rho+v \nabla^{2} \boldsymbol{u}-\alpha \boldsymbol{u}-(\phi \nabla \mu)-A \boldsymbol{g}+\boldsymbol{f},
$$

The text following Eq. (2) should read as "where $P$ is pressure and $\rho(=1)$ is the constant density, together with the incompressibility...." The paper has been corrected as of 12 January 2017. The equation and text are correct in the printed version of the journal. 